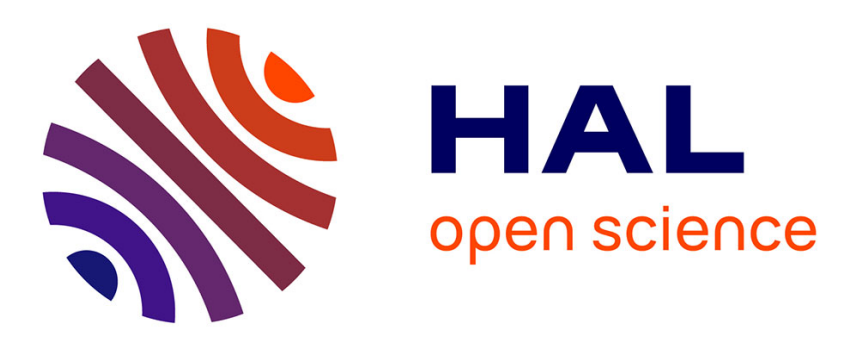

\title{
Solvent-Assisted Fractionation of Oligomeric Cellulose and Reversible Transformation of Cellulose II and IV
}

Xin Zhang, Feng Jiang, Cesar Torres-Luna, Yoshiharu Nishiyama, Robert M Briber, Howard Wang

\section{- To cite this version:}

Xin Zhang, Feng Jiang, Cesar Torres-Luna, Yoshiharu Nishiyama, Robert M Briber, et al.. SolventAssisted Fractionation of Oligomeric Cellulose and Reversible Transformation of Cellulose II and IV. ACS Biomaterials Science and Engineering, 2021, 7 (10), pp.4792-4797. 10.1021/acsbiomaterials.1c00885 . hal-03374861

\section{HAL Id: hal-03374861 \\ https://hal.univ-grenoble-alpes.fr/hal-03374861}

Submitted on 12 Oct 2021

HAL is a multi-disciplinary open access archive for the deposit and dissemination of scientific research documents, whether they are published or not. The documents may come from teaching and research institutions in France or abroad, or from public or private research centers.
L'archive ouverte pluridisciplinaire HAL, est destinée au dépôt et à la diffusion de documents scientifiques de niveau recherche, publiés ou non, émanant des établissements d'enseignement et de recherche français ou étrangers, des laboratoires publics ou privés. 


\section{Solvent-Assisted Fractionation of Oligomeric Cellulose and Reversible Transformation of Cellulose II and IV}

Xin Zhang, ${ }^{\dagger, \#}$ Feng Jiang, ${ }^{\dagger, \star, \neq}$ Cesar Torres, ${ }^{\dagger}$ Yoshiharu Nishiyama, ${ }^{\dagger}$ Robert M. Briber, ${ }^{\dagger}$ and Howard Wang ${ }^{\dagger, \text {,** }}$

'Department of Materials Science and Engineering, University of Maryland, College Park, MD 20742, USA

${ }^{\star}$ Biomass Molecular Engineering Center, Department of Materials Science and Engineering, School of Forestry and Landscape Architecture, Anhui Agricultural University, Hefei, Anhui 230036, China

"Univ. Grenoble Alpes, CNRS, CERMAV, 38000 Grenoble, France

${ }^{\perp}$ Neutron Science Platform, Songshan Lake Materials Laboratory, Dongguan, Guangdong 523808, China

\#These authors contributed equally to this work

*Corresponding author

Email:wangh@umd.edu; wangh@ @slab.org.cn 
ABSTRACT: Oligomeric cellulose with an average degree of polymerization of 7.68 and a polydispersity of 1.04 has been fractionated using solution processes. Three fractions have been obtained through initial dissolution, subsequent crystallization, and solvent precipitation, respectively. The resulting oligocellulose fraction has an average degree of polymerization of 7.70 and polydispersity of 1.01 , respectively. Cellulose $\mathrm{IV}_{2}$ crystals form in the oligocellulose fraction, and reversibly transform to II and back to IV using simple solvents. 


\section{INTRODUCTION}

Cellulose is the most abundant organic substance on Earth, and knowledge on its structures and behaviors has advanced tremendously over the last two centuries. Cellulose occurs in nature as high molecular weight semicrystalline polymers that exhibits high strength and durability, good thermal stability, and excellent biocompatibility and biodegradability, ${ }^{1,2}$ and finds wide applications from conventional structural materials to novel functional materials in membranes, electronics, elastomers, foams, hydrogels and aerogels. ${ }^{3-7}$ However, the full potential of cellulose properties is yet to be explored. While most applications use cellulose in native states with high degree of polymerization (DP), oligocellulose of monodisperse size is one of model systems for better understanding the physical chemistry of cellulose and for developing novel cellulosic

materials as a building block. ${ }^{15-17}$ Particularly in life science applications, oligocellulose can find use as food-additives such as sweetener and dietary fiber for weight control and disease prevention, and as biomedical materials such as bio-surfactants, bioelastomers, adhesives and hydrogels. ${ }^{9-13}$

Various methods have been developed for preparing oligocellulose, such as chemical and enzymatic synthesis, enzymatic and acidic hydrolysis, as well as thermal, mechanical and hybrid methods, ${ }^{8}$ whereas a reliable and scalable routine for producing monodisperse oligocellulose in large quantities is still lacking. In a previous study, we revisited the classical acidic hydrolysis preparation of oligomeric cellulose at room temperature, ${ }^{14}$ and characterized the lower DP fraction, DP7, which is composed of oligocellulose with DP's ranging from 5 to 12. In this study, DP7 is further fractionated through initial dissolution in dimethyl sulfoxide (DMSO), subsequent crystallization, and solvent precipitation, respectively. The obtained three fractions were 
characterized using Fourier-transform infrared (FTIR), nuclear magnetic resonance (NMR), Xray diffraction (XRD), matrix-assisted laser desorption/ionization time-of-flight mass spectrometry (MALDI-TOF MS), small angle neutron scattering (SANS), cross-polarized optical microscopy (OM), differential scanning calorimetry (DSC), scanning electron microscopy (SEM), and atom force microscopy (AFM) to reveal their chemical and physical natures.

\section{EXPERIMENTAL SECTION}

Materials. Microcrystalline cellulose (MCC, Avicel PH-101) and phosphoric acid (85\% by mass aqueous solution) were purchased from Sigma-Aldrich. Other analytical-grade chemical reagents were commercially available and used as received. The starting oligocellulose fraction DP7 having a weight-averaged DP of 7.68, and a polydispersity index, PDI $=1.04$, was prepared according to the reported method. ${ }^{14,18}$ In this study, DP7 has been further fractionated to yield three fractions, denoted as F1, F2 and F3, in a process as follows. DP7 powder was added to DMSO at $5 \%$ mass ratio and stirred at $90{ }^{\circ} \mathrm{C}$ for an hour. The non-soluble fraction was collected by centrifuging at $5000 \mathrm{rpm}$ for $10 \mathrm{~min}$ at room temperature and thoroughly washed with acetone, and vacuumed dried to yield the fraction F3. The supernatant was kept at $15{ }^{\circ} \mathrm{C}$ for 3 hours; solid precipitates formed and were collected by centrifugation. The remaining solution went through a freeze-thaw cycle between $-20{ }^{\circ} \mathrm{C}$ and room temperature; solids from the process were collected by centrifugation. The solids from isothermal storage at $15{ }^{\circ} \mathrm{C}$ and freeze-thaw processes gave the same XRD patterns, and together yielded the fraction F2. The supernatant after the freeze-thaw cycle was precipitated in 5 times volume of acetone. The precipitates were collected by centrifuge, cleaned by re-dispersion and centrifugation in acetone for 5 times, and 
dried in vacuum oven at room temperature. The resulting white powder is the fraction F1. The yield of $\mathrm{F} 1$ is ca. $10 \%$ of the mass of the starting DP7.

Characterizations. Nicolet Nexus 670 FTIR spectrometer was used to record the spectra of samples. NMR spectra were recorded on a Bruker Avance 400 NMR spectrometer. XRD patterns were recorded using a Xenocs Xeuss with a micro-focusing $\mathrm{Cu} \mathrm{K} \alpha$ source and a highresolution area detector. Bruker Autoflex Speed MALDI-TOF MS was used with 2,5dihydroxybenzoic acid as the substrate material to determine the molecular mass of each fraction. SANS measurements were carried out on the NGB-30 m SANS at the NIST Center for Neutron Research (NCNR), National Institute of Standards and Technology (NIST). SEM and AFM measurements were conducted on Hitachi SU70 field emission SEM and a Cypher ES Environmental AFM, respectively. Olympus XB-70 cross-polarized microscope was used to observe the spherulite crystals. TA Instruments DSC Q100 was used to determine the melting point of the cellulose-solvent complex.

\section{RESULTS AND DISCUSSION}

Following the data analysis routine reported previously, ${ }^{14}$ MALDI-TOF spectra yield the DP histogram of the DP7 and fractions F1-F3 as shown in Fig. 1a. DP7 contains molecules with DPs from 4 to 12, centering at 7. F3 has DPs from 6 to 12, with the peak at 8, implying dissolution of low DP molecules in DMSO. F3 has the average DP of 8.59 and PDI of 1.03. F2 from the crystalline complex with DMSO has an average DP of 9.96 and PDI of 1.03, respectively. F1 
contains predominantly DP7 (42.8 mol\%) and DP8 (37.0 mol\%), flanked by DP6 (7.0 mol\%) and DP9 $(10.5 \mathrm{~mol} \%) . \mathrm{F} 1$ has DP $=7.70$ and PDI $=1.01$, and is the target product of the solution fractionation process. Crystalline structures of cellulose fractions were analyzed using XRD. Circularly averaged 1D spectra are shown in Fig. 1b. DP7 shows a typical cellulose II structure; F2 shows unique cellulose-DMSO co-crystal structures not previously reported, which is the topic of a separate study; F1 resembles features of cellulose IV, as will be discussed below.
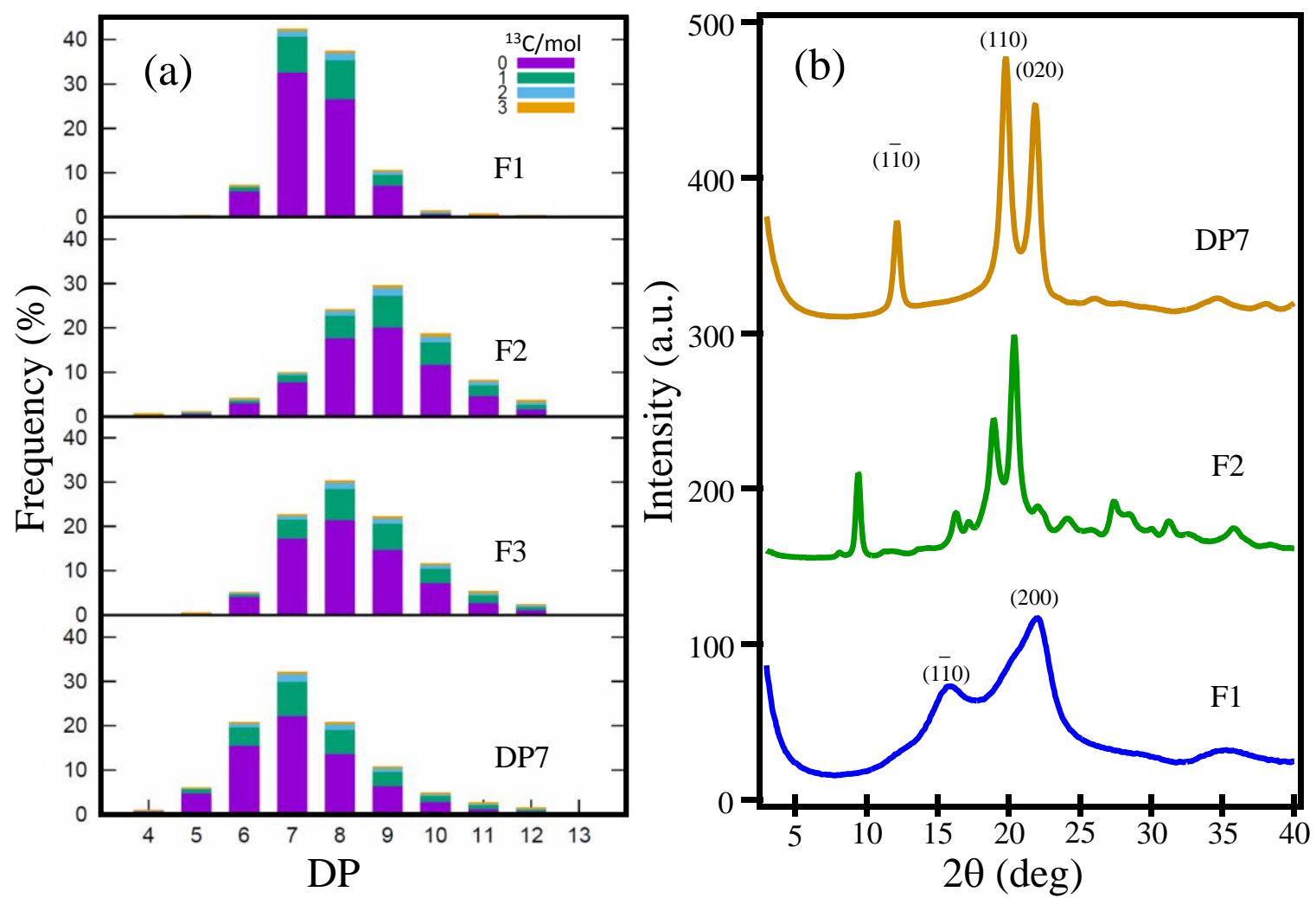

Figure 1. (a) Histograms of molecular weight distribution of oligocellulose fractions, from bottom to top, DP7 the starting material, F3 the non-soluble gel faction, F2 the DP7-DMSO cocrystallite fraction, and F1 the DMSO-soluble and acetone-precipitated fraction; color coding indicating the number of ${ }^{13} \mathrm{C}$ atoms in a molecule. (b) Powder XRD patterns of, from top to 
bottom, DP7 in cellulose II structure, F2 the DP7-DMSO complex crystals, and F1 the acetone precipitates with cellulose IV features.

The morphology of DP7 has been studied using microscopy and scattering techniques. SANS on $1 \mathrm{wt} \%$ DP7 in $\mathrm{D}_{2} \mathrm{O}$ and deuterated DMSO (dDMSO) was measured at room temperature. Figure 2a shows that with comparable contrast factors the scattering intensity of the DP7 aqueous suspension (green) is about tenfold higher than the DMSO one (blue), implying strong DP7 aggregation in water, or solubilization of fraction of DP7 in DMSO. The $Q^{-2}$ power-law scattering over a wide range indicates a dominant 2D morphology, and the slightly higher power exponent at low $Q$ suggests sheet bending and stacking. SEM micrograph in Fig. 2b on DP7 dried from aqueous suspension shows stacking of crumpled sheets. A drop of dilute DP7 aqueous suspension was cast on a clean silicon wafer; the surface topology was examined using an Asylum Research Cypher ES AFM with a TESP-V2 tip. Figure 2c shows mostly a monolayer coverage of the substrate surface with submicron-size irregular-shaped DP7 lamellae, while the height profile in Fig. 2d indicates their thickness of ca. $5 \mathrm{~nm}$, implying extended chain crystals. 

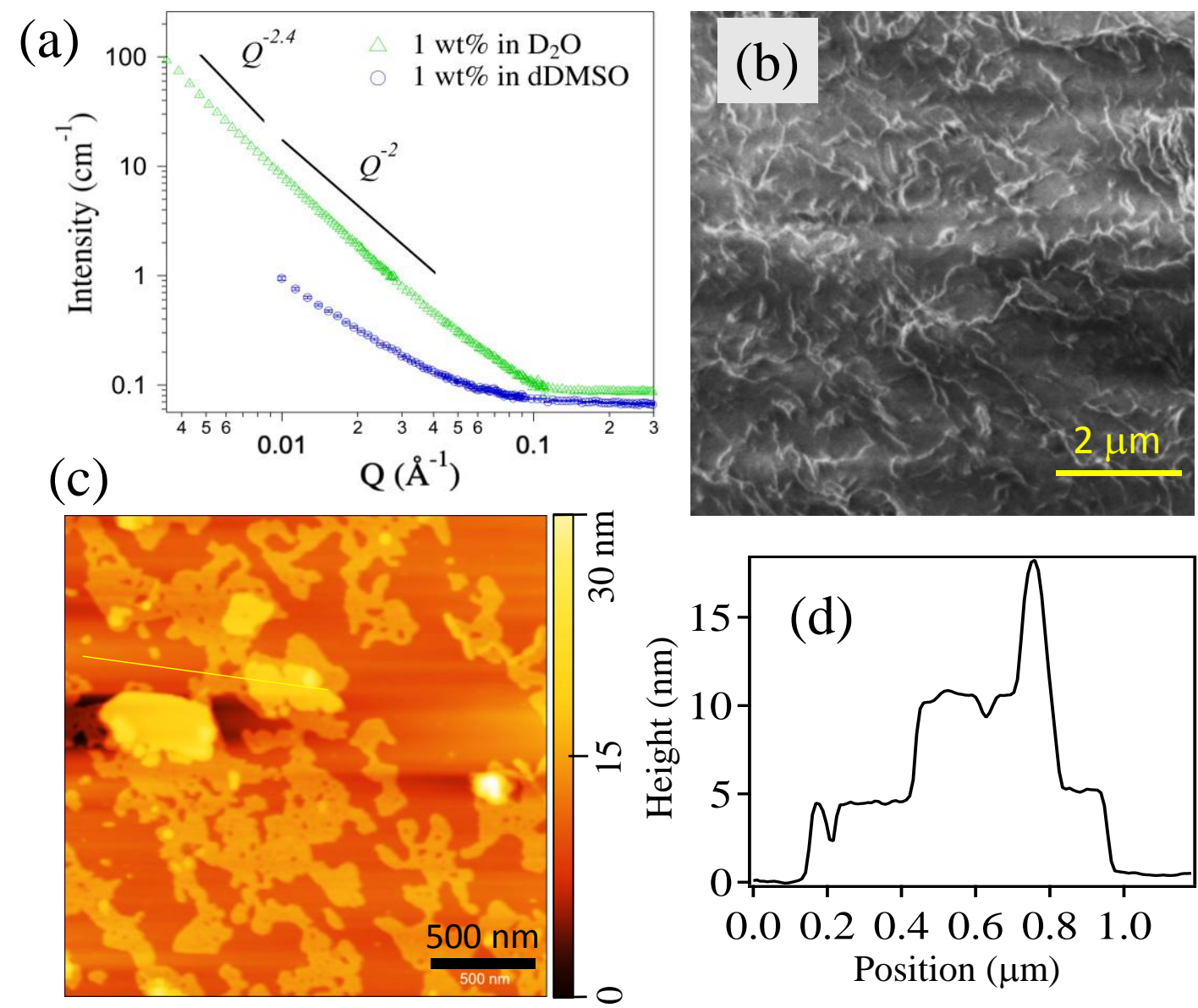

Figure 2. (a) SANS of $1 \mathrm{wt} \% \mathrm{DP} 7$ in $\mathrm{D}_{2} \mathrm{O}$ (green) and deuterated DMSO (blue), respectively. High scattering intensity of the former implying strong DP7 aggregation; the $Q^{-2}$ power law over a wide range implying dominantly 2D structures. (b) SEM on dried DP7 from water suspension showing crumpled sheet-like features. (c) AFM on DP7 crystallites casting from dilute suspension on silicon wafer, showing monolayer of submicron-size irregular lamellae. (d) Height profiles along the line through multilayered stacks in (c) indicating a lamellar thickness of ca. 5 nm. 
The morphology of F2, the fraction crystalline in DMSO, is shown in Fig. 3. After storing the DMSO solution with $2 \mathrm{wt} \% \mathrm{DP} 7$ at $15{ }^{\circ} \mathrm{C}$ for 9 hours, a drop of solution was sealed in a square capillary tube and examined using cross-polarized microscope. A bright field optical micrograph shows spherulites of ca. $40 \mu \mathrm{m}$ in diameter in Fig. 3a, where the one in focus shows concentric rings with fine textures. Figures $3 b$ and $3 c$ are cross-polarized micrographs of the same spherulites revealing the banding structure, while the latter with the insertion of a retardation plate shows blue second and fourth quadrants, indicating crystallites of the negative birefringence, where the chain axis is tangential to the spherulite. This kind of birefringence is commonly found in semicrystalline polymers where the crystal grows perpendicular to the chain direction often forming lamellar crystals of folded chains. Cellulose oligomers usually form negative spherulites, however positive spherulite crystals from cellulose have also been reported for enzymatic synthesis in water-acetonitrile mixtures, in which chains might be along radial direction during rapid crystal growth. ${ }^{19,20}$ Also, cellulose-ionic liquid complex shows positive spherulite, but the chain direction is tangential, and the birefringence is inversed due to the orientation of ionic liquid molecules. ${ }^{19,20}$ Spherulites were cast on a silicon substrate in a dry glovebox to prevent water condensation. A specimen free of conductive layer coating was imaged using a low voltage electron beam at $700 \mathrm{~V}$ acceleration voltage in a Hitachi SU70 field emission SEM. Figure 3d shows a compact spherulite of ca. $20 \mu \mathrm{m}$ in diameter with a relatively smooth surface. The internal structure of a spherulite revealed on a microtomed film of spherulite-embedded epoxy resin is shown in Fig. 3e. The likely nucleation site is indicated by an arrow, and edge-on and flat-on lamellae are marked with " $e$ " and " $f$ ", respectively, implying lamellae twisting while growing outward. The repeating distance of $e$ and $f$ is ca. $5 \mu \mathrm{m}$, consistent with the banding periodicity in Figs. 3a-3c. To view individual lamellae, spherulites in 
acetone dispersion was sonicated, and a drop of supernatant was cast on silicon wafer in a glovebox. AFM micrograph in Fig. 3f shows a large flat lamella extending over micrometers, with some holes. The depth of the hole indicates a lamellar thickness of ca. $5 \mathrm{~nm}$, as obtained from a line scan profile (Fig. 3g) across a hole in the lamella. The thickness is close to the chain length of DP9, implying extended-chain crystals of oligocellulose. F2 is sensitive to water vapor, a brief exposure transforms the complex to cellulose II crystals. DSC scan in Fig. S3 shows F2 melting at $\sim 50{ }^{\circ} \mathrm{C}$ in DMSO. 


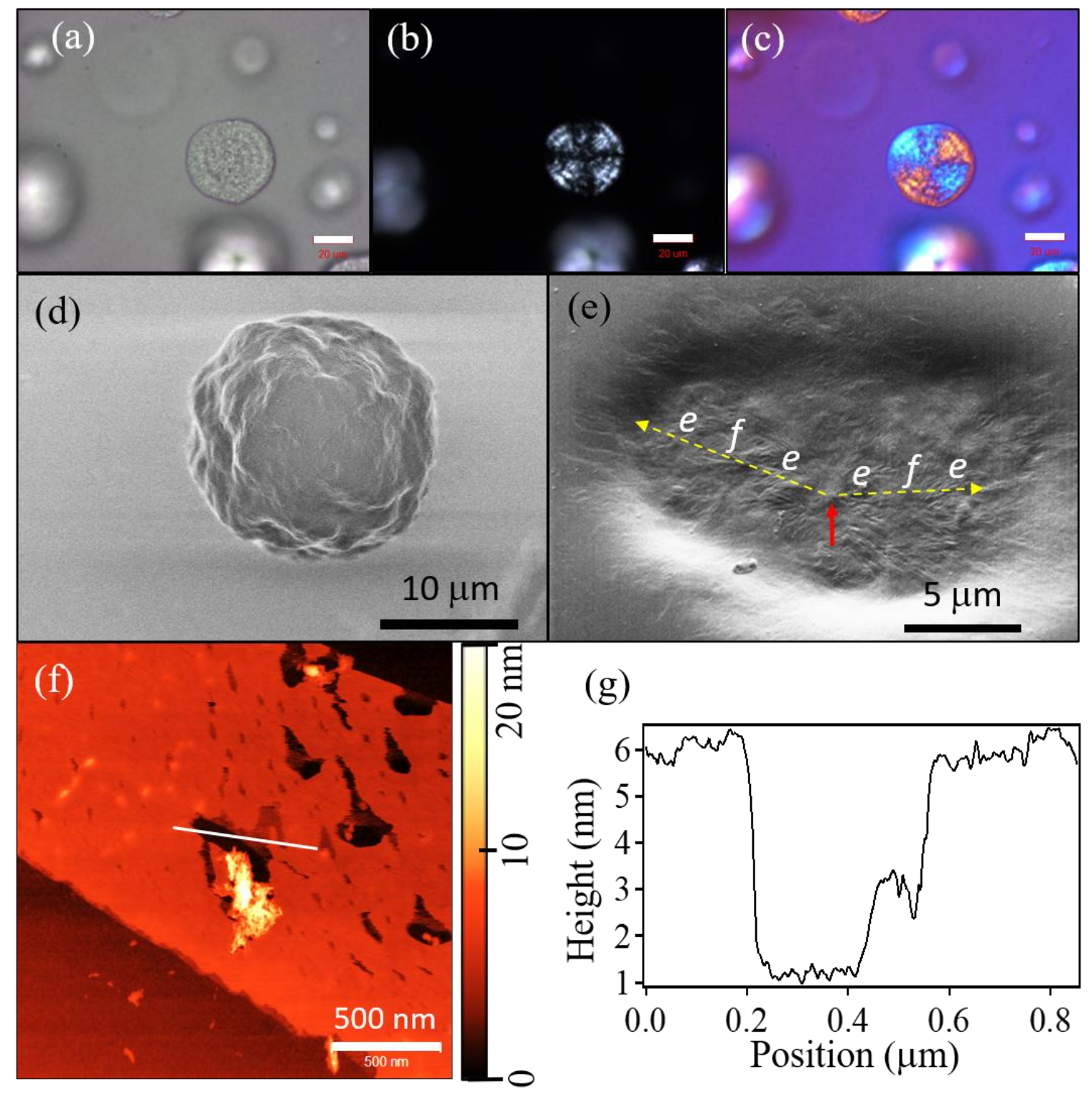

Figure 3. Optical micrographs of banded spherulites of F2 in DMSO solution using (a) bright field, (b) cross polarized, and (c) Gamma-plate-inserted cross polarized contrasts, respectively, showing negative spherulites with banding periodicity of ca. $5 \mu \mathrm{m}$. Scale bars $20 \mu \mathrm{m}$; (d) SEM micrographs of a F2 spherulite in the surface view. (e) SEM on the cross-section view reveals regularly twisting lamellar stacking as growing from the nucleation center, indicated by the red arrow, and edge-on (labelled ' $e$ ") and flat-on (labelled " $f$ ') morphologies of lamellae, 
respectively. (f) AFM on a lamella lying flat on a silicon wafer showing crystallites of microns in lateral dimension and nanometers in thickness; $(\mathrm{g})$ the height profile along the line in (f) indicating lamellar thickness of ca. $5 \mathrm{~nm}$.

AFM micrographs of the cold DMSO soluble fraction precipitated in acetone (F1 fraction) and drop-cast on silicon substrate from acetone suspension after thorough wash were shown in Fig. 4a. Cellulose aggregates appear as irregular flakes with lateral dimensions from 10's nm up to micrometers and relatively uniform thickness of a few nanometers. The insets of $400 \mathrm{~nm} \times 400$ $\mathrm{nm}$ graphs show details of two distinct features at local areas. The flat valley regions are mostly covered with monolayer lamellae as shown in the lower inset, like those observed in Figs. 2c and 3f, whereas hills appear crumpled and fibril-like, resembling aggregated needles as shown in the upper inset. The line scan in Fig. $4 \mathrm{~b}$ shows the height of constituent objects to be about $6 \mathrm{~nm}$. F1 transforms between cellulose IV and II with the assistance of solvents. F1 as-collected from acetone precipitation (F1-0) transforms to cellulose II upon immersing in water for a few seconds (F1-1), as shown in Fig. 4c. Upon exposing to saturated DMSO vapor at $100{ }^{\circ} \mathrm{C}$ in a sealed vial for 12 hours, F1-1 transforms to mostly cellulose $\mathrm{IV}_{2}$, denoted as F1-2 in Fig. 4c, in which a weak peak at ca. $12^{\circ}$ implies co-existing cellulose II. The diffraction peaks of F1-2 are sharper than those of F1-0, while F1-2 similarly transforms to mostly cellulose II upon soaking in water (F1-3). Note that data in Fig. 4c were obtained on the same piece of F1 specimen of $1 \mathrm{~mm} \times 1$ $\mathrm{mm}$ in lateral size to minimize sample variation. In comparison, the cellulose II of DP7 and water treated F2 was not altered upon exposing to DMSO vapor at $100{ }^{\circ} \mathrm{C}$. 

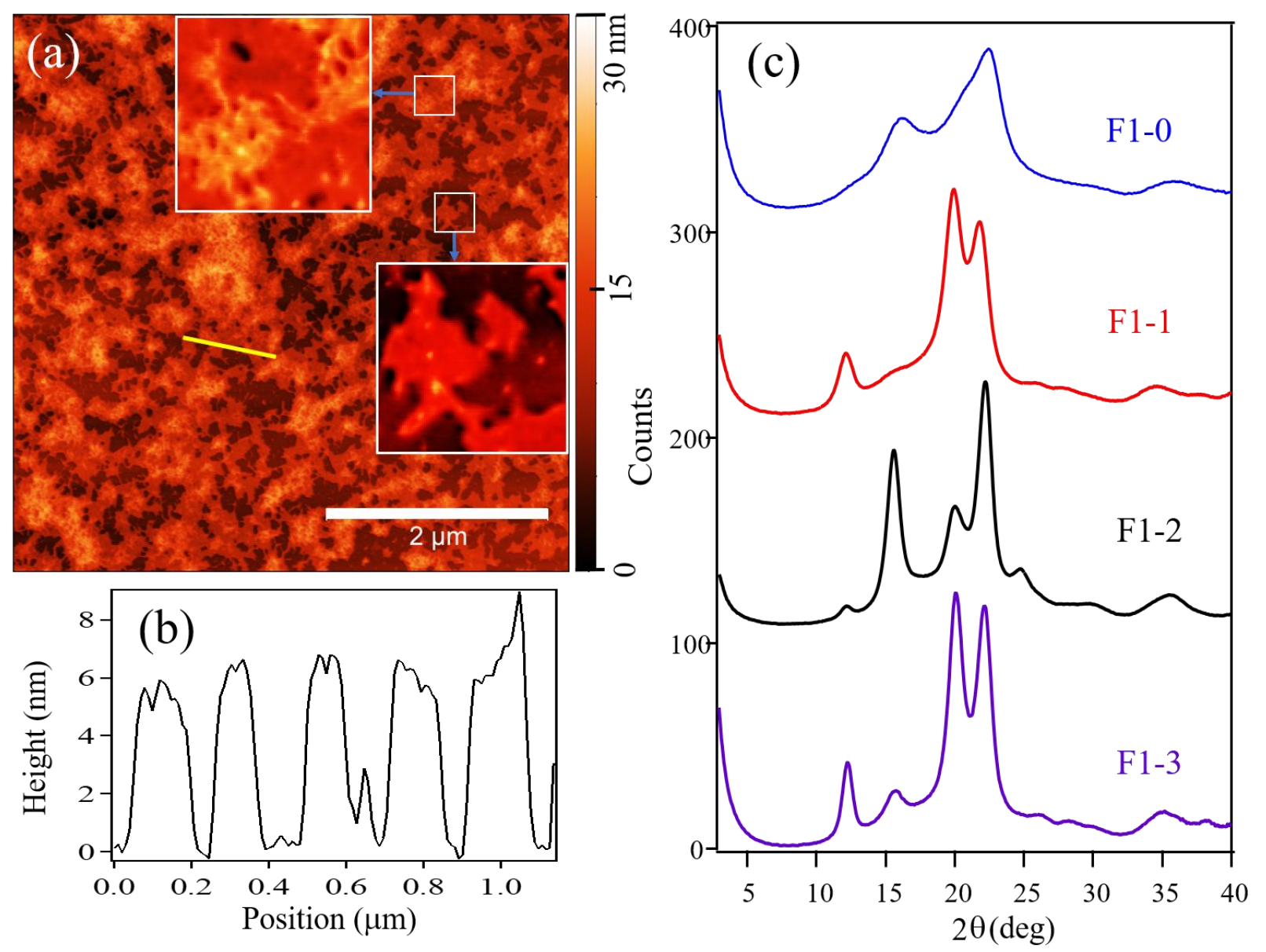

Figure 4. Morphology of F1. (a) AFM showing irregular flakes of cellulose aggregates with lateral dimensions from tens nm up to microns and relatively uniform thickness of a few nanometers. The insets of $400 \mathrm{~nm} \times 400 \mathrm{~nm}$ graphs show details at local areas of valley with lamellae (lower) and hills with fibril (upper) features, respectively. (b) The height profile along the yellow line in (a) indicating flake thicknesses of ca. $6 \mathrm{~nm}$. (c) XRD showing the structural evolution of F1: F1-0 the as-prepared with cellulose IV features, F1-1 after soaking in water cellulose IV turned into II; F1-2 upon exposing to hot DMSO vapor cellulose II transformed to $\mathrm{IV}_{2}, \mathrm{~F} 1-3$ upon soaking in water transforms back to Cellulose II. 
Cellulose $\mathrm{IV}_{2}$ would form by heating cellulose $\mathrm{III}_{2}$ crystal in glycerol at $260{ }^{\circ} \mathrm{C}$, as was initially reported in 1941 and reviewed in later literatures. ${ }^{21,22}$ Cellulose $\mathrm{IV}_{2}$ has also been produced by regenerating cellulose xanthate at $100{ }^{\circ} \mathrm{C}$ in the fiber form, ${ }^{23}$ and heating DP21 cellulose II in water at $190{ }^{\circ} \mathrm{C}$ for 2 hours. ${ }^{24}$ Others reported the formation of cellulose $\mathrm{IV}_{2}$ via deacetylation of low-DP cellulose triacetate and water-precipitation, treating DP18 with methylamine and DMSO mixtures, ${ }^{25}$ and supercritical or compressed water hydrolysis. ${ }^{26,27}$ The examples shows that cellulose IV is a high-temperature phase obtained from relatively loose or activated high-energy structures. Similarly in this study, solution precipitation in acetone, although at room temperature, is a kinetic-controlled process resulting in fine structures of high-energy states. The evolution of fine structures during the precipitation and subsequent acetone washing could be responsible for the variety of morphology as shown in Fig. 4a, which is a topic of future studies.

Narrow molecular weight distribution has also been found to affect the crystal morphology, such that DP24 at PDI $=1.11$ producing more perfect crystals. ${ }^{28}$ In another study, enzymatically synthesized oligocellulose with an average DP of 9 form highly ordered cellulose II. ${ }^{29}$ Comparing to previous findings, this study demonstrates new pathways of interconversion between cellulose IV and II by simple solvent environment in an oligocellulose fraction with a lower DP at 7.70 and narrower polydispersity at 1.01. As low DP oligocellulose forms extended chain crystals and ensures surface energetics not insignificant comparing to bulk quantities, it is conceivable that hydrogen bonding of DMSO at the surface or in the bulk of thin crystals plays a critical role in the structure transformation, either through a transient state or in the final structure. Data imply that DMSO molecules reside in the bulk in F2 while stay at surface in F1, consistent with their corresponding PDI's. 


\section{CONCLUSIONS}

In summary, oligomeric cellulose with an averaged degree of polymerization of 7.68 and a polydispersity of 1.04 has been fractionated via selective precipitation processes. Three fractions have been produced, through initial dissolution, subsequent crystallization, and solvent

precipitation, sequentially. The resulting DMSO soluble cellulose fraction has a weight averaged degree of polymerization of 7.70 and polydispersity of 1.01 . Formation of cellulose $\mathrm{IV}_{2}$ crystals have been observed in the near-monodisperse fraction, which is to the best of our knowledge the lowest DP cellulose forming IV structures. This study also reports two alternative pathways for producing cellulose IV crystal, through precipitating DMSO solution in acetone and through exposing cellulose II to hot DMSO vapor, respectively, as well as reversible transformation between cellulose II and IV crystals using facile solvent processes.

\section{ASSOCIATED CONTENT}

\section{Supporting Information}

The Supporting Information is available free of charge on the ACS Publications website at https://pubs.acs.org/doi/.

FTIR spectra of F1-0, F1-1 specimens, with cellulose II, dry DMSO and wet DMSO; ${ }^{1} \mathrm{H}$ NMR spectra of DP7, glucose and cellobiose; DSC heat flow curve of F2 suspension in DMSO (PDF). 


\section{AUTHOR INFORMATION}

\section{Corresponding Author}

*E-mail: wangh@umd.edu; wangh@sslab.org.cn.

\section{Notes}

The authors declare no competing financial interest.

\section{ACKNOWLEDGMENTS}

We acknowledge the support of the National Institute of Standards and Technology through award 70NANB12H238 and by providing the neutron research facilities used in this study. F. Jiang was financially supported by the National Natural Science Foundation of China (Grant 51603199) and Anhui Provincial Innovation and Entrepreneurship Support Plan for Overseas Returnees (Grant 2020LCX027). We thank Dr. Yimin Mao of NCNR/NIST for help on SANS experiment and data reduction. We acknowledge the support of the Maryland NanoCenter and its AIMLab.

\section{REFERENCES}

(1) Garcia-Valdez, O.; Champagne, P.; Cunningham, M. F. Graft Modification of Natural Polysaccharides via Reversible Deactivation Radical Polymerization. Prog. Polym. Sci. 2018, 76, 151-173. 
(2) Jiang, F.; Pan, C.; Zhang, Y.; Fang, Y. Cellulose Graft Copolymers toward Strong Thermoplastic Elastomers via RAFT Polymerization. Appl. Surf. Sci. 2019, 480, 162-171.

(3) Jiang, F.; Wang, F.; Pan, C.; Fang, Y. Cellulose-Based Thermoplastics and Elastomers via Controlled Radical Polymerization. In Thermosoftening Plastics, IntechOpen, 2019, pp. 35-51.

(4) Ajdary, R.; Tardy, B. L.; Mattos, B. D.; Bai, L.; Rojas, O. J. Plant Nanomaterials and Inspiration from Nature: Water Interactions and Hierarchically Structured Hydrogels. Adv. Mater. 2020, 2001085.

(5) Pang, B.; Jiang, G.; Zhou, J.; Zhu, Y.; Cheng, W.; Zhao, D.; Wang, K.; Xu, G.; Yu, H. Molecular-Scale Design of Cellulose-Based Functional Materials for Flexible Electronic Devices. Adv. Electron. Mater. 2021, 7, 2000944.

(6) Heise, K.; Kontturi, E.; Allahverdiyeva, Y.; Tammelin, T.; Linder, M. B.; Ikkala, O. Nanocellulose: Recent Fundamental Advances and Emerging Biological and Biomimicking Applications. Adv. Mater. 2021, 33, 2004349.

(7) Lamm, M. E.; Li, K.; Qian, J.; Wang, L.; Lavoine, N.; Newman, R.; Gardner, D. J.; Li, T.; Hu, L.; Ragauskas, A. J. Recent Advances in Functional Materials through Cellulose Nanofiber Templating. Adv. Mater. 2021, 33, 2005538.

(8) Billès, E.; Coma, V.; Peruch, F.; Grelier, S. Water-Soluble Cellulose Oligomer Production by Chemical and Enzymatic Synthesis: A Mini-Review. Polym. Int. 2017, 66, 1227-1236. 
(9) Kamitakahara, H.; Nakatsubo, F.; Klemm, D. New Class of Carbohydrate-Based Nonionic Surfactants: Diblock Co-Oligomers of Tri-O-Methylated and Unmodified CelloOligosaccharides. Cellulose 2007, 14, 513-528.

(10) Enomoto-Rogers, Y.; Kamitakahara, H.; Yoshinaga, A.; Takano, T. Radially Oriented Cellulose Triacetate Chains on Gold Nanoparticles. Cellulose 2010, 17, 923-936.

(11) Nasiri, M.; Reineke, T. M. Sustainable Glucose-Based Block Copolymers Exhibit Elastomeric and Adhesive Behavior. Polym. Chem. 2016, 7, 5233-5240.

(12) Hata, Y.; Kojima, T.; Koizumi, T.; Okura, H.; Sakai, T.; Sawada, T.; Serizawa, T. Enzymatic Synthesis of Cellulose Oligomer Hydrogels Composed of Crystalline Nanoribbon Networks under Macromolecular Crowding Conditions. ACS Macro Lett. 2017, 6, 165-170.

(13) Adharis, A.; Petrović, D. M.; Özdamar, I.; Woortman, A. J.; Loos, K. Environmentally Friendly Pathways Towards the Synthesis of Vinyl-Based Oligocelluloses. Carbohydr. Polym. 2018, 193, 196-204.

(14) Jiang, F.; Zhang, X.; Hwang, W.; Nishiyama, Y.; Briber, R. M.; Wang, H. Oligocellulose from Acid Hydrolysis: A Revisit. Appl. Surf. Sci. 2021, 537, 147783.

(15) Gupta, R.; Lee, Y. Mechanism of Cellulase Reaction on Pure Cellulosic Substrates. Biotechnol. Bioeng. 2009, 102, 1570-1581.

(16) Zweckmair, T.; Oberlerchner, J. T.; Böhmdorfer, S.; Bacher, M.; Sauerland, V.; Rosenau, T.; Potthast, A. Preparation and Analytical Characterisation of Pure Fractions of Cellooligosaccharides. J. Chromatogr. A 2016, 1431, 47-54. 
(17) Billès, E.; Onwukamike, K. N.; Coma, V.; Grelier, S.; Peruch, F. Cellulose Oligomers

Production and Separation for the Synthesis of New Fully Bio-Based Amphiphilic Compounds. Carbohydr. Polym. 2016, 154, 121-128.

(18) Isogai, A.; Usuda, M. Preparation of Low-Molecular-Weight Cellulose Using PhosphoricAcid. Mokuzai Gakkaishi 1991, 37, 339-344.

(19) Kobayashi, S.; Hobson, L. J.; Sakamoto, J.; Kimura, S.; Sugiyama, J.; Imai, T.; Itoh, T. Formation and Structure of Artificial Cellulose Spherulites via Enzymatic Polymerization. Biomacromolecules 2000, 1, 168-173.

(20) Song, H. Z.; Niu, Y. H.; Yu, J.; Zhang, J.; Wang, Z. G.; He, J. S. Preparation and Morphology of Different Types of Cellulose Spherulites from Concentrated Cellulose Ionic Liquid Solutions. Soft Matter 2013, 9, 3013-3020.

(21) Osullivan, A. C. Cellulose: The Structure Slowly Unravels. Cellulose 1997, 4, 173-207. (22) Zugenmaier, P. Crystalline Cellulose and Cellulose Derivatives: Characterization and Structures. Springer-Verlag: Berlin Heidelberg, 2008; Chapter 2, pp 7-27.

(23) Gardiner, E. S.; Sarko, A. Packing Analysis of Carbohydrates and Polysaccharides .16. The Crystal-Strucures of Cellulose-IV I and Cellulose-IV II. Can. J. Chem. 1985, 63, 173-180.

(24) Isogai, A.; Usuda, M.; Kato, T.; Uryu, T.; Atalla, R. H. Solid-State CP/Mas Carbon-13 NMR Study of Cellulose Polymorphs. Macromolecules 1989, 22, 3168-3172.

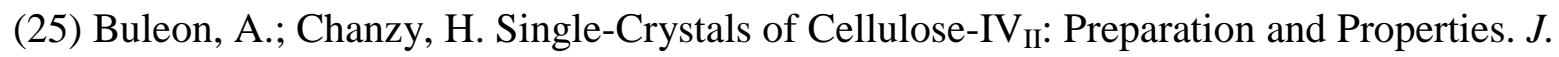
Polym. Sci., Part B: Polym. Phys. 1980, 18, 1209-1217. 
(26) Abdullah, R.; Ueda, K.; Saka, S. Decomposition Behaviors of Various Crystalline

Celluloses as Treated by Semi-Flow Hot-Compressed Water. Cellulose 2013, 20, 2321-2333.

(27) Buffiere, J.; Abad, N.; Ahvenainen, P.; Dou, J. Z.; Cocero, M. J.; Sixta, H. Tailoring the Structure and Morphology of Low-Molecular-Weight Cellulose Produced During Supercritical Water Hydrolysis. ACS Sustain. Chem. Eng. 2018, 6, 16959-16967.

(28) Buleon, A.; Chanzy, H.; Froment, P. Single-Crystals of Cellulose-IV Cellulose Molecular-Weight. J. Polym. Sci., Part B: Polym. Phys. 1982, 20, 1081-1088.

(29) Hiraishi, M.; Igarashi, K.; Kimura, S.; Wada, M.; Kitaoka, M.; Samejima, M. Synthesis of Highly Ordered Cellulose II in Vitro Using Cellodextrin Phosphorylase. Carbohydr. Res. 2009, 344, 2468-2473. 
Table of Contents

Solvent-Assisted Fractionation of Oligomeric Cellulose and Reversible Transformation of Cellulose II and IV

Xin Zhang, ${ }^{\dagger, \#}$ Feng Jiang, ${ }^{\dagger, \$, \#}$ Cesar Torres, ${ }^{\dagger}$ Yoshiharu Nishiyama, ${ }^{\prime}$ Robert M. Briber, ${ }^{\dagger}$ and Howard Wang ${ }^{\dagger, \perp, *}$

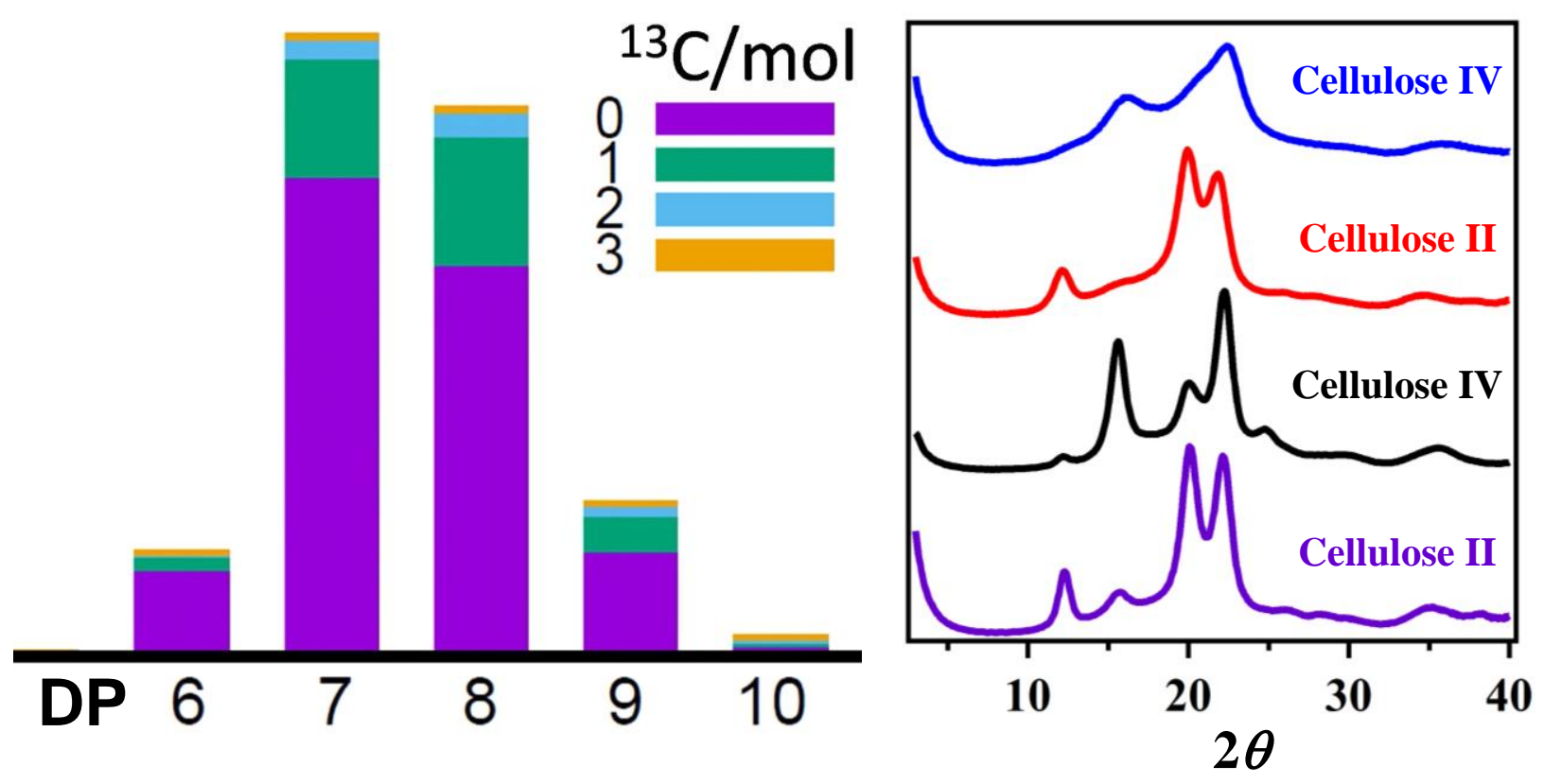

